\title{
In Vitro Release of Isoforms of Prolactin from Pituitary Glands of Turkey Hens at Different Physiological Stages
}

\author{
G. Bédécarrats, D. Guémené, C. Morvan, S. Crisóstomo-Pinto, U. Kühnlein and D. Zadworny
}

\section{Introduction}

In turkey hens, changes in blood and pituitary content of prolactin (PRL) are known to occur during a reproductive cycle. Low levels are associated with sexually immature, out-of-lay and moulting hens, increasing levels occur during egg laying, and high levels are associated with incubation behaviour. In avian species vasoactive intestinal peptide (VIP) is recognised as the major PRL releasing factor in vivo as well as in vitro.

Among vertebrates, PRL is present as several molecular isoforms whose ratio fluctuates with age and physiological and pathological states. In humans, the different isoforms of PRL are secreted through different pathways. Similarly in rats, heterogeneity in form and function of lactotrophs has been reported and the different subpopulations of cells secrete different PRL variants. In the turkey hen, the transition from egg laying to incubation behaviour is associated with a significant increase in both lactotroph and mammosomatotroph cells. Further more, in turkeys, PRL is present in the pituitary gland in 3 different isoforms; 1 nonglycosylated (NG-) and 2 glycosylated (G-). The ratio between $\mathrm{G}$ and NG-PRL isoforms in pituitary gland has been shown to fluctuate during a reproductive cycle with NG-PRL being the predominant isoform in out-of-lay and moulting hens and the G-PRL isoforms being predominant in laying and incubating hens. Currently, no information on the type and the ratio of PRL isoforms released by the pituitary gland have been reported.

\section{Objective}

In the present study, we assessed the type of isoforms of PRL released by the pituitary from turkey hens at different physiological stages using an in vitro perifusion procedure. We also assessed the type of isoforms released during cVIP stimulation.

\section{Experimental procedures Tissue source}

Medium white turkey hens were raised under standard conditions and maintained on a $6 \mathrm{~h}$ light photoperiod until 30 weeks of age when sexual activity was photoinduced by extending the photoperiod to $14 \mathrm{~h}$ light. Food and water were provided ad libitum. Seven different physiological stages ( $n=4$ per stage) were studied: layers which were laying eggs for less than 8 weeks (L8), for 12 to 15 weeks (L12) and for over 24 weeks (L24); hens which were incubating for less than 4 weeks (I4) and for over 10 weeks (I10); Out-of-lay hens (OL) and moulting hens (Mo). Eggs were collected during four daily inspections (09:00, 11:30, 14:00, 16:30). At the time of the egg collections, the presence of a hen in the nest was recorded. A hen was considered as incubating if it was sitting (in or outside the nestbox) at least 3 times out of 4 daily inspections during a minimum of 3 consecutive days (spending more than $75 \%$ of its time nesting). A hen was considered as out-of-lay when it ceased laying for more than 3 weeks and did not show incubation behaviour or visible signs of spontaneous moulting. The status of the hen was then confirmed by rectal palpation. The moulting hens were force moulted by submitting them to a $6 \mathrm{~h}$ light photoperiod 6 weeks prior to pituitary collection. The animals were killed by cervical dislocation and the anterior pituitaries were collected within 2 minutes of death.

\section{Perifusion system}

The glands were cut longitudinally into 2 hemi-pituitaries immediately after collection and placed into icecold M199 culture medium. Each hemi-pituitary was then weighed, diced into small pieces and put into $0.5 \mathrm{ml}$ perifusion chambers (Acusyst. S, Endotronics) containing M199 standard medium with phenol red (Sigma Chemical). The M199 medium was supplemented with glucose $(2.0 \mathrm{~g} / \mathrm{l})$, amphotericin B (5 $\mathrm{mg} / \mathrm{ml})$, gentamicin $(0.1 \% \mathrm{w} / \mathrm{v})$, sodium bicarbonate $(0.35 \mathrm{~g} / \mathrm{l})$ and HEPES $(10 \mathrm{mM})$. The chambers were continuously perifused with $0.25 \mathrm{ml} / \mathrm{min} \mathrm{M} 199$ at $39^{\circ} \mathrm{C}$ and gassed with a $95 \% \mathrm{O}_{2} / 5 \% \mathrm{CO}_{2}$ mix. Eight perifusion chambers were used simultaneously and the samples were perifused $(0.25 \mathrm{ml} / \mathrm{min})$ for $2 \mathrm{~h}$ before data collection started. The perifused media was collected in fractions of $5 \mathrm{~min}$ ( $1.25 \mathrm{ml} /$ fraction) and stored at $-20^{\circ} \mathrm{C}$ until assayed. 
Based on a time course previously assessed in preliminary experiments, a 2 min stimulation with cVIP at a concentration of $10^{-7} \mathrm{M}$ (Sigma Chemical) previously diluted in M199 was performed 30 min after the collection started.

\section{Radioimmunoassay}

The concentration of PRL was measured in triplicate on 10 to 100 $\mu \mathrm{l}$ aliquots of perifusate using a specific tPRL radio-immuno-assay (RIA). The PRL content was expressed as $\mathrm{ng} / \mathrm{ml}$ of perifusate/mg of tissue. A mean basal release value was calculated for each chamber on the 6 samples collected before cVIP stimulation.

\section{Western-blotting procedure}

After VIP stimulation, $40 \mu l$ aliquots of the fractions from individual hemipituitaries were directly analysed by western-blotting. As the levels of PRL were too low to be detected in individual samples before cVIP stimulation for most of the perifusion chambers, pools were made for each physiological stage with $75 \mu \mathrm{l}$ aliquots of each chamber $(8 \times 75=600 \mu \mathrm{l})$. The volume was then reduced to $50 \mu \mathrm{l}$ with a Centricon 10 concentrator (Amicon, Inc.) and analysed by westernblotting.

The samples were separated on $12 \%$ SDS polyacrylamide gels. A broad range prestained protein marker (New England Biolabs Inc.) was introduced in each gel. The proteins were electrotransfered onto PVFD membrane (Immobilon-P, $0.45 \mu \mathrm{m}$, Millipore) at constant voltage of 100 $\mathrm{V}$ for $1 \mathrm{~h} 30 \mathrm{~min}$ at $4{ }^{\circ} \mathrm{C}$ with constant stirring in $25 \mathrm{mM}$ Tris, 192 mM glycine, $20 \%$ methanol, $\mathrm{pH} 8.3$. The membranes were blocked in Tris buffered saline $\mathrm{pH} 7.5$ (TBS) 5\% non fat dry milk and incubated overnight with rabbit anti recombinant turkey prolactin antibody at a 1/2000 dilution in TBS $0.5 \%$ non fat dry milk. The membranes were washed 4 times with TBS $0.5 \%$ Tween 20 , incubated $1 \mathrm{~h}$ with $10^{6} \mathrm{cpm} /$ membrane $^{125} \mathrm{I}$ radiolabelled protein $A$ (specific activity > $30 \mu \mathrm{Ci} / \mu \mathrm{g}$; ICN Biomedicals), washed 4 times with TBS $0.05 \%$ Tween 20 and finally exposed to Kodak X-ray film for autoradiography at different time of exposure. The proportion of each immunoreactive band was calculated by densitometric analyses using GP Tools 3.0 software (BioPhotonics Corporation) after digitalisation of the X-ray film.

\section{Statistical Analysis}

All statistical analyses were done with Statview (ABACUS Concept inc.) on Macintosh. Data were compared using a factorial ANOVA and a Fisher protected least significant difference (PLSD) test if appropriate ( $\mathrm{p}<0.05$; ANOVA).

\section{Results and discussion}

The time course of the release of PRL by pituitary glands obtained from turkey hens at different physiological stages is presented on Figure 1. All the hemi-pituitaries responded to cVIP stimulation by significantly $\quad(\mathrm{p}<0.05) \quad$ increasing their release of PRL. The response was observed within 5 to $10 \mathrm{~min}$ after stimulation, with a maximum obtained on average $15 \mathrm{~min}$ after stimulation. The highest amplitude was observed for the I4 group whereas the lowest was observed for the Mo group.

Typical western blotting analyses of individual perifusion chambers from hemipituitaries from turkey hens at different physiological stages are presented in Figure 2. The western blotting analysis revealed two immunoreactive bands with a relative molecular weight of 27 and $24 \mathrm{kDa}$ which migrated at the same position as previously described in pituitary extract (Bédécarrats et al., Research Report 1995). The analysis by western blotting of the pooled samples from the basal release of PRL by pituitary glands from turkey hens at different physiological stages is presented in Figure 3. The percentage of $27 \mathrm{kDa}$ band for $\mathrm{L} 8$, L12, L24, I4, I10, OL and Mo groups were 70, 69, 58, 70, 71, 46 and $40 \%$, respectively. The percentage of $27 \mathrm{kDa}$ observed by western blotting following cVIP stimulation is presented in Figure 4. The post cVIP stimulation time course was divided into 3 sections, from 0 to 5,10 to 20 and 20 to 40 min after stimulation. No significant differences were found in the percentage of $27 \mathrm{kDa}$ isoform among the 3 intervals for any physiological stages studied. The overall percentage of $27 \mathrm{kDa}$ immunoreactive band in perifusate was significantly higher $(\mathrm{p}<0.01)$ for the L8 I4 and I10 groups and significantly $(\mathrm{p}<0.01)$ lower for the Mo group.

The two isoforms detected are consistent with the G- and NG-PRL isoforms previously described in literature with the $27 \mathrm{kDa}$ and 24 $\mathrm{kDa}$ corresponding to the G- and NG-PRL, respectively. In pituitary extracts from turkey hens, about $70 \%$ and $60 \%$ of the PRL was GPRL in incubating and laying hens, respectively, whereas about $38 \%$ and $33 \%$ of the PRL was G-PRL in outof-lay and moulting hens, respectively (Bédécarrats et al., Research Report 1995). In the perifusion system, the PRL isoforms are released in approximately the same ratio as was observed in pituitary extracts at corresponding 
physiological stages. Thus, it is likely that both G- and NG-PRL are present in the blood of turkeys. In human prolactinoma cells G- and NG-PRL are secreted through a constitutive and a controlled after storage pathway, respectively. In rats, different subpopulations of mammotrophs having different secretion pathways for PRL have been detected, and the subpopulations release different types of PRL variants. Although the secretion of PRL by the pituitary gland is under an inhibitory control in mammals, whereas it is under a stimulatory control in the turkey, it is unlikely that the different isoforms of PRL synthetized by the pituitary are released via constitutive and controlled pathways. In chicken, the expression of PRL gene can be induced in different areas of the pituitary gland when stimulated with VIP or growth hormone releasing factor. In turkey hens, a difference exists in the distribution of pituicytes in the adenohypophysis between laying and incubating hens. Lactotrophs are present only in the cephalic lobe in laying and somatotrophs are replaced by lactotrophs in the ventral half of the caudal lobe in pituitaries from incubating hens. This replacement is progressive and a transition mammosomatotroph cell type synthesizing PRL and growth hormone can be observed. Moreover, the transcription level of the PRL gene as well as the stablity of the PRL mRNA change with the photoperiod and the physiological status of turkey hens. Since the distribution of the lactotrophs, the transcription of the PRL gene, the stability of PRL mRNA as well as the ratio G- versus NG-PRL are related to the physiological status of the hen, a possible relation between pituicyte population, the abundance and the stability of PRL mRNA and the synthesis and the release of different PRL variants needs to be investigated.

As previously reported in vivo as well as in vitro, VIP appeared in our perifusion system to be a potent PRL releasing factor on pituitary glands from hens at different physiological stages. The cVIP stimulation did not result in any changes in the ratio of isoforms released by the perifused hemipituitary. Specific VIP membrane receptors have been found in the anterior pituitary gland from turkey hens in higher levels in incubating and lower levels in nonphotostimulated and photorefractory hens. Thus, the presence of higher relative amounts of G-PRL in the pituitary gland from incubating hens combined with an increased VIP stimulation, probably leads to the presence of high levels of G-PRL in the blood. As VIP was reported to stimulate the transcription of the PRL gene in cultured pituitary cells within $24-48 \mathrm{~h}$, it is possible that VIP may act on the posttranslational modification of PRL, although we did not investigate the effect of VIP on de novo synthesis of PRL.

Glycosylation of protein hormones has been shown to change their half life as well as modify the binding activity to its receptor. In the turkey, G-PRL has lower binding activity than NG-PRL to its receptor and in addition, appears to be less bioactive in stimulating casein production in mammary gland explants. It is therefore interesting that G-PRL is dominant during the hyperprolactinemic state associated with incubation behaviour in galliforms and NG-PRL is dominant during out of lay stages of the reproductive cycle (Bédécarrats $e t$ al., Research Report 1995). Since the major role attributed to PRL in the turkey is initiation and maintenance of incubation, G-PRL is most likely the variant involved in this behaviour. Whether or not, in turkey hens, the variants of PRL differ in their metabolic clearance rate or binding to a putative binding protein in the blood circulation or to its receptor on target tissues still needs to be clarified. However, the results of our study may provide a mechanism to partition the biological effects of PRL to specific tissues according to the ratio of isoforms. In support of the latter it is notable that in the human, glycosylation of PRL differentially down-regulates its actions at individual target tissues, facilitates proteolytic cleavage which results in PRLs with different biological activities which act through their own unique receptors and that the ratio of PRL variants change during physiological states. It is also worth noting that glycosylation is thought to modulate protein folding and stability which would in turn potentially affect interaction with its receptor. In this regard, the consensus sequence for N-linked glycosylation of tPRL (Asn - X - Cys at positions 56 and 197) is located at sites which could potentially affect the secondary structure associated with disulfide bond formation, thus leading to altered receptor affinity and biological activity.

\section{Impact}

In vertebrates, PRL is known to be synthesised as a number of molecular variants whose ratio fluctuates with different physiological and pathological states. In the current study, we provide strong evidence that the PRL variants which are synthesized in the pituitary gland of turkey hens (Bédécarrats et al., Research Report 1995) are secreted in the same 
proportion. Since greater than 300 separate actions have been attributed to PRL, it is likely that posttranslational modification of PRL would modulate its activities. However, although PRL variants in large number of species have been identified which vary with physiological state, further studies are required to clarify the specific roles of these isoforms.

\section{Acknowledgements}

Funding support from the Institut National de la Recherche Agronomique (INRA) and the Natural Science and Engineering Research Council of Canada (NSERC) are gratefully acknowledged. The cooperation of BETINA (56250 St Noff, Elven, France) in providing turkey hens was greatly appreciated. Our thanks to M. Garreau-Mills, S. Nevoit for their technical assistance.

\section{Figure legends}

\section{Figure 1:}

Evolution in the concentrations of prolactin ( $\mathrm{ng} / \mathrm{min} / \mathrm{mg}$ of pituitary) present in perifusate from hemipituitaries of turkey hens at different physiological stages stimulated with cVIP at a concentration of $10^{-7} \mathrm{M}$ for $2 \mathrm{~min}$ (mean \pm SEM). $\leftarrow$ represents the time of cVIP stimulation.

\section{Figure 2:}

Typical SDS PAGEs followed by western blots of perifusate from single hemipituitaries of turkey hens at different physiological stages (L8, L12, L24, I4, I10, O1, Mo) around a cVIP stimulation at a concentration of $10^{-7} \mathrm{M}$ for $2 \mathrm{~min}$. Lane 1 to 8 represents the time course of perifusion with: lane 1, before cVIP; lane 2, 0 to $5 \mathrm{~min}$ after cVIP; lane 3, 5 to 10 min after cVIP; lane 4,10 to
15 min after cVIP; lane 5, 20 to 25 min after cVIP; lane 6,35 to $40 \mathrm{~min}$ after cVIP; lane 7,45 to 50 min after cVIP; and lane 8, 65 to $70 \mathrm{~min}$ after cVIP.

\section{Figure 3:}

SDS PAGE followed by western blotting of pooled samples from basal release perifusate from hemipituitaries of turkey hens at different physiological stages (I4, L8, L12, L24, O1, Mo, I10).

\section{Figure 4:}

Percentage of total turkey PRL as 27 $\mathrm{kDa}$ PRL immunoreactive band measured by densitometry after western blotting detection of perifusate from hemipituitaries of turkey hens at different physiological stages following cVIP stimulation.

\section{Figure 1:}

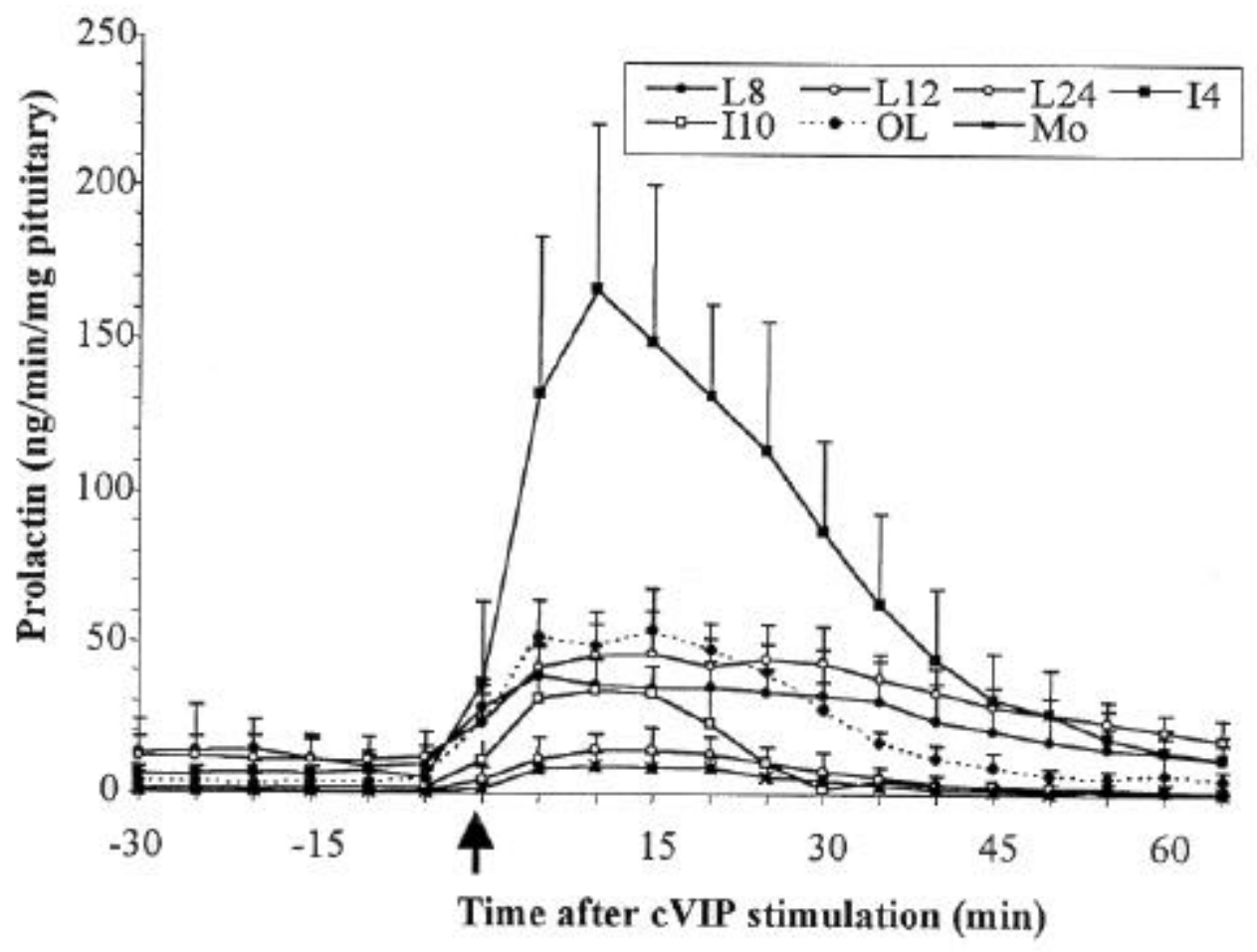


Figure 2:

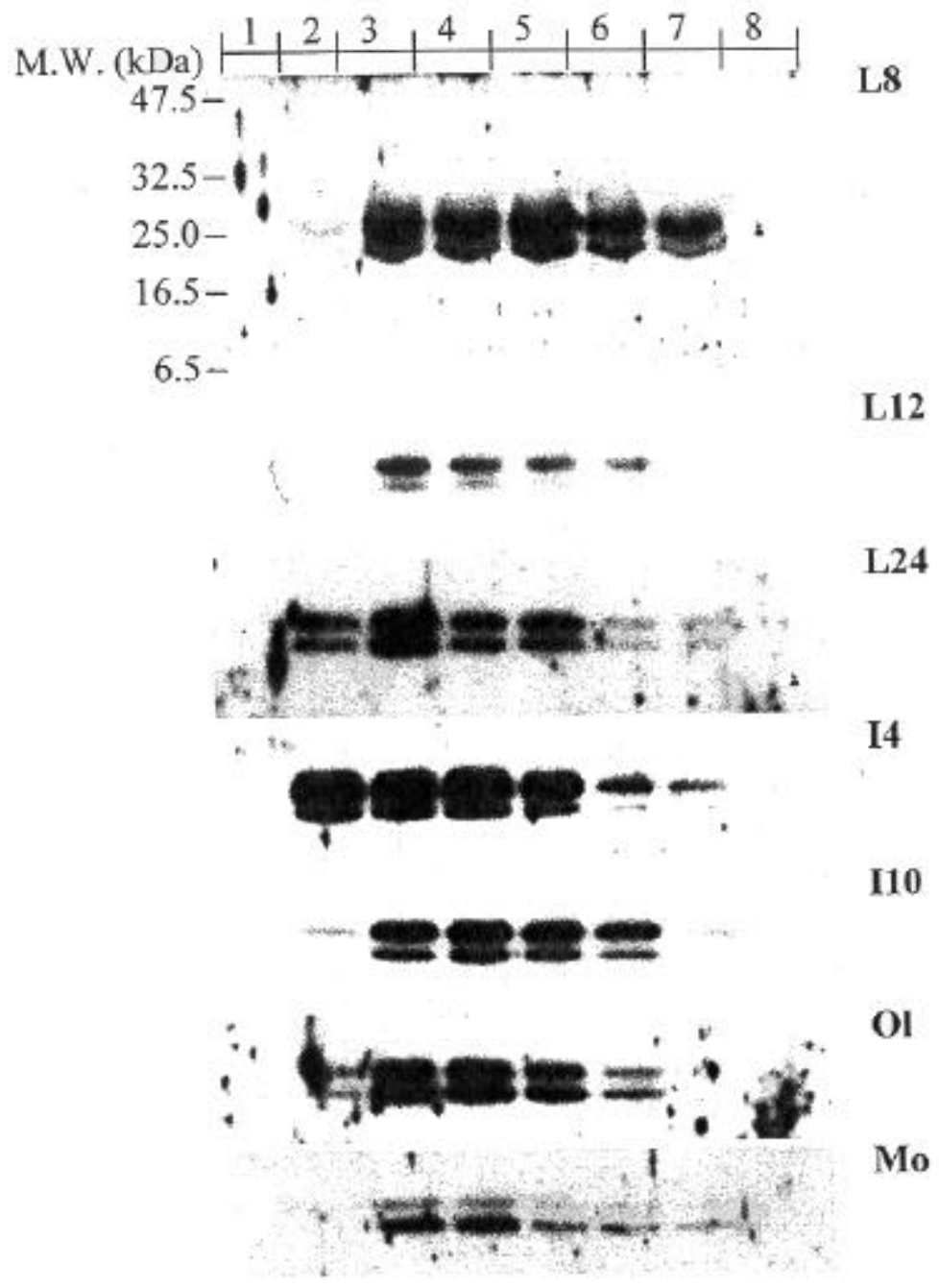

Figure 3:

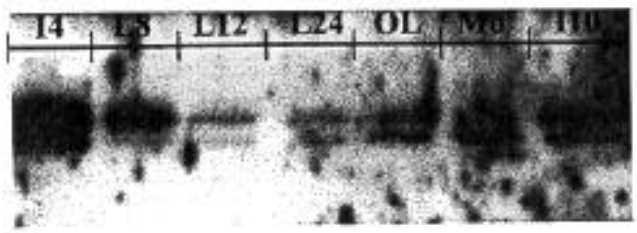

